\title{
On CCD standard stars and flat-field calibration
}

\author{
J. Manfroid ${ }^{\star}$ \\ Institut d'Astrophysique, Université de Liège, avenue de Cointe 5, B-4000 Liège, Belgium
}

Received September 21, 1995; accepted January 19, 1996

\begin{abstract}
Numerical simulations of a CCD flat-fielding calibration procedure on stellar fields have been performed in the monochromatic case. The relations between the photometric conditions, the observational procedure and the calibration accuracy are explored. This analysis shows how and why one must establish high-quality standard sequences in clusters or moderately crowded fields. The advantages of a long-term management of the reduction procedures is stressed.
\end{abstract}

Key words: instrumentation: detectors — methods: data analysis — methods: observational — techniques: image processing - techniques: photometric

\section{Introduction}

Systematic use of cluster standard stars instead of isolated standards in CCD photometry has two advantages:

- It is obviously more efficient to pack hundreds of standard stars in a few frames than observing a dozen isolated standards. A wide diversity of stellar types, classes and reddenings can be achieved by an adequate selection.

- As a bonus, cluster standards provide the lowfrequency component of the flat-field calibration of the detector - including the color terms and, if needed, a shutter-timing correction - , as shown in a previous paper (Manfroid 1995, Paper I);

Common sense and a bit of experimentation can lead to an efficient observational strategy in order to i) set up cluster standards and ii) to use them efficiently. However, accurate results can only be obtained with an appropriate, rigorous, calibration procedure.

The mathematical method developed in Paper I is such a procedure. It considerably improves the photometric calibration of CCD images and is ideal for establishing a system of cluster standards as well as for using them in photometric reductions.

The methods normally used to derive flat-field calibration (e.g., night-sky stacks) do not properly address the case of stellar objects, and they do not give color terms. The zero points - and the color transformations - obtained with isolated standards are valid for only a small area of the CCD and errors of several percents may arise at

${ }^{\star}$ Research Director, Belgian Fund for Scientific Research (FNRS) other locations (see, e.g., Tobin 1993). The procedure discussed in Paper I leads to a straightforward simultaneous solution to these problems, as well as an easy determination of shutter-timing effects by tying the low spatialfrequency calibration to actual stellar measurements, i.e., to point sources instead of extended ones.

As discussed in Paper I, there is a strong coupling between the zero points derived for each frame and the linear spatial components of the flat-field calibration. This may explain why such procedures did not receive the full attention they deserve. It is often easy to eliminate this coupling. If the magnitudes of the stars are unknown - e.g., when defining a new system - , at least part of the observations (as few as three exposures) must be conducted in perfect photometric conditions. If a sufficient number of stars have accurate magnitudes in the natural system, the coupling is lifted and the calibration can be carried out even in non-photometric conditions.

In order to support these conclusions and to evaluate the accuracy of the method proposed in Paper I under various observing conditions, we conducted extensive numerical simulations. These lead us to define efficient observational strategies.

\section{Numerical simulation}

We mostly use the same notations as in Paper I: ${ }^{1}$

$-\boldsymbol{x}(s)=(x(s), y(s))$ is the position of star $s$ on the CCD;

- $m_{0}(f, s, \boldsymbol{x})$ is the instrumental magnitude of star $s$ calculated from frame $f$;

\footnotetext{
${ }^{1}$ Note that boldface enhancement for vectors $\boldsymbol{x}$ and $\boldsymbol{b}$ was not correctly rendered in the printed version of Paper I.
} 
- $m(s)$ is the actual magnitude of star $s$;

We simulate monochromatic observations of a moderately crowded field with randomly distributed stars with an average density $n_{\mathrm{s}}$ stars per frame. Successive frames are taken with offsets in $x$ or $y$ coordinates. The unit offsets $(\delta x, 0)$ and $(0, \delta y)$ have an amplitude of $1 / 5$ th the side of the CCD (assumed to be square). A spiraling pattern allows to cover an arbitrary large area of the star field.

Each frame has a specific zero point which may represent the observing conditions. We define a systematic component $z_{\mathrm{gr}}(g)$ valid for a whole group $g$ of $n_{\mathrm{fr}}(g)$ frames obtained in similar conditions and a random component $z_{\mathrm{fr}}(f)$ of variance $\sigma_{\mathrm{fr}}^{2}(g)$ which reflects short-term variations of the sky occuring between frames.

Frames within a particular group $g_{0}$ may have been obtained in exceptionally good photometric conditions and present a particularly small $\sigma_{\mathrm{fr}}\left(g_{0}\right)$.

In addition to this, each frame has the same low spatial-frequency component $z_{\mathrm{ff}}(\boldsymbol{x})$, i.e., we suppose that the high-frequency component has been fully corrected.

Each star measurement is affected by some noise $z_{\mathrm{m}}(f, s)$ of variance $\sigma_{\mathrm{s}}^{2}$. We assume a simple dependency of the noise versus magnitude, in such a way that there is a uniform distribution $\sigma_{\mathrm{s}, 1}<=\sigma_{\mathrm{s}}<=\sigma_{\mathrm{s}, 2}$.

We can then write:

$$
m_{0}(f, s, \boldsymbol{x})=m(s)+z_{\mathrm{gr}}(g)+z_{\mathrm{fr}}(f)+z_{\mathrm{ff}}(\boldsymbol{x})+z_{\mathrm{m}}(f, s) .
$$

Table 1 presents a sample of 21 cases among the infinity of possible variations. For each of them we performed about 500 totally independent simulations. One or two parameters take a few discrete values, so their effect can be accurately analyzed. Simulations include a maximum of 16 exposures. In most cases, we assumed the stars to be reasonably bright, so they all have the same observational noise $\sigma_{\mathrm{s}, 1}=\sigma_{\mathrm{s}, 2}$.

\subsection{Reductions}

When necessary, the frames are grouped the same way as they are built, i.e., we assume we can distinguish periods of different photometric conditions (bad weather, very different air masses, etc.).

The model that must be solved is described by Eq. (3) of Paper I. The parameters $a(g)$ are zero-point frame corrections. For the function $\Psi(\boldsymbol{x} ; \boldsymbol{b})$ we choose a thirdorder polynomial in $x$ and $y$ with parameters $\boldsymbol{b}=\left\{b_{i}\right\}$ $(i=1, \ldots, 9)$, i.e., we do not consider the effects of a possible mismatch between the true flat-field correction $z_{\mathrm{ff}}$ and the mathematical model. In practice, simple polynomials appear to be reasonable approximations to the smooth low-frequency flat-field corrections. This could be expected from vignetting effects as well as from a major part of the scattering. Other functions - smooth or not - $\Psi(\boldsymbol{x} ; \boldsymbol{b})$ and $z_{\mathrm{ff}}(\boldsymbol{x})$ involving a similar number of parameters would give the same results as the present simulations. Non-smooth, realistic, model functions can be build as linear combinations of empirical look-up tables derived by observing the variations of flat fields obtained in extremely different conditions.

Reductions are carried out in the same way as actual observations, i.e., the parameters $a(g), b(i)$ - and $m(s)$ if star $s$ is not standard - are determined with our regular photometric reduction programme which makes use of the optimization technique described in Paper I.

Table 1. Log of simulated observations. The sigmas are quoted in $0.001 \mathrm{mag}$. Free parameters are indicated by the '-' symbol

\begin{tabular}{|c|c|c|c|c|c|c|c|c|c|}
\hline Fig. & $n_{\mathrm{s}}$ & $n_{\mathrm{gr}}$ & $n_{\mathrm{fr}}$ & $n_{\mathrm{fr}}\left(g_{0}\right)$ & $\sigma_{\mathrm{s}, 1}$ & $\sigma_{\mathrm{s}, 2}$ & $\sigma_{\mathrm{fr}}$ & $\sigma_{\mathrm{fr}}\left(g_{0}\right)$ & $z_{\mathrm{gr}}$ \\
\hline 1 & 22 & 1 & 16 & 0 & - & $\sigma_{\mathrm{s}, 1}$ & 2 & 0 & 0 \\
\hline 2 & 22 & 1 & 16 & 0 & - & $\sigma_{\mathrm{s}, 1}$ & 8 & 0 & 0 \\
\hline 3 & 22 & 1 & 16 & 0 & - & $\sigma_{\mathrm{s}, 1}$ & 32 & 0 & 0 \\
\hline 4 & 22 & - & $16 / n_{\mathrm{gr}}$ & 0 & 10 & 10 & 4 & 0 & 0 \\
\hline 5 & 22 & $16-n_{\mathrm{fr}}\left(g_{0}\right)$ & 1 & - & 10 & 10 & 32 & 4 & 0 \\
\hline 6 & 22 & - & 1 & 3 & 10 & 10 & 32 & 4 & 0 \\
\hline 7 & 22 & 1 & - & 0 & 4 & 4 & 4 & 0 & 0 \\
\hline 8 & 22 & 1 & - & 0 & 8 & 8 & 4 & 0 & 0 \\
\hline 9 & - & 1 & 8 & 0 & 4 & 4 & 4 & 0 & 0 \\
\hline 10 & 22 & 1 & 16 & 0 & 4 & - & 4 & 0 & 0 \\
\hline 11 & 22 & 4 & 4 & 0 & 4 & 12 & 4 & 0 & - \\
\hline 12 & 22 & 16 & 1 & 3 & 10 & 10 & 8 & 8 & 0 \\
\hline 13 & 22 & 1 & 16 & 0 & - & $\sigma_{\mathrm{s}, 1}$ & 2 & 0 & 0 \\
\hline 14 & 22 & 1 & 16 & 0 & - & $\sigma_{\mathrm{s}, 1}$ & 32 & 0 & 0 \\
\hline 15 & 22 & - & $16 / n_{\mathrm{gr}}$ & 0 & 10 & 10 & 4 & 0 & 0 \\
\hline 16 & 22 & - & $16 / n_{\mathrm{gr}}$ & 0 & 10 & 10 & 32 & 0 & 0 \\
\hline 17 & 22 & 1 & - & 0 & 4 & 4 & 4 & 0 & 0 \\
\hline 18 & 89 & 1 & - & 0 & 4 & 4 & 4 & 0 & 0 \\
\hline 19 & - & 1 & 8 & 0 & 4 & 4 & 4 & 0 & 0 \\
\hline 20 & 22 & 1 & 16 & 0 & 4 & - & 4 & 0 & 0 \\
\hline 21 & 22 & 4 & 4 & 0 & 4 & 12 & 4 & 0 & - \\
\hline
\end{tabular}

\section{Discussion}

Figures 1 to 21 show the median absolute deviation (MAD) (over the whole CCD area) between the true and the calculated flat-field corrections (in 0.001 magnitude) for the 21 cases of Table 1 The 95th percentile (AD95) is also plotted. Regression lines are superimposed in order to provide a quick visual estimate of the general trends.

\subsection{No standard stars}

Figures 1 to 3 show the effect of the measurement accuracy $\sigma_{\mathrm{s}}$ under, respectively, perfect, average, and mediocre photometric conditions (i.e., for increasing $\sigma_{\mathrm{fr}}$ ). All 16 frames are grouped together. This is the only possibility when magnitudes are unknown and when there was no obvious period of outstanding photometric conditions. It is clear that good overall photometric conditions are needed to reach $\mathrm{AD} 95<0.01$.

Blindly fractioning the frames into smaller groups degrades the results, as shown in Fig. 4. The situation 


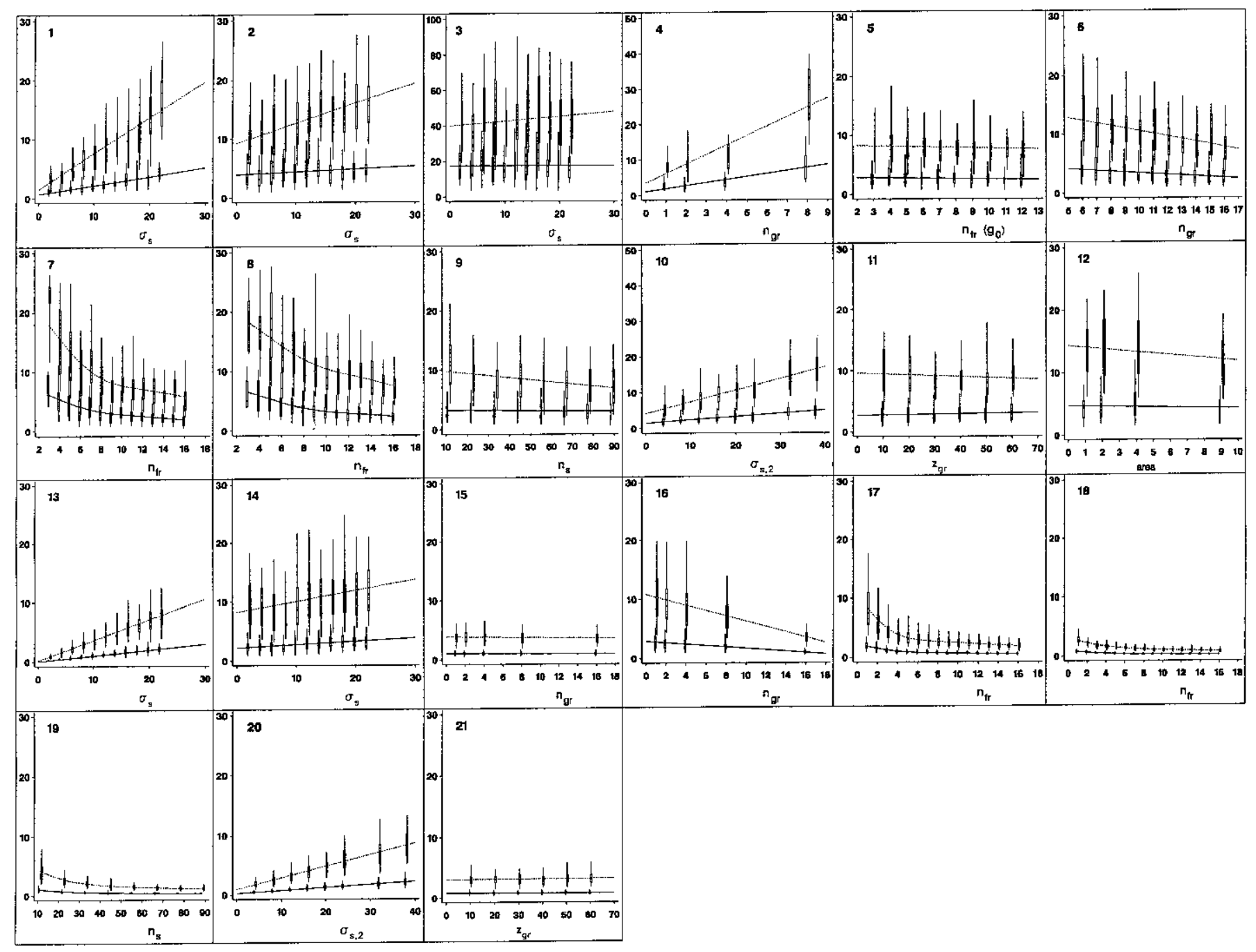

Fig. 1. -21. Absolute deviation of the calculated flat-field correction when there are no standard stars (Figs. 1-12), and when they are present (Figs. 13-21). Series of about 50 individual measurements are combined and displayed as two box-and-whisker symbols: the lower symbol is the Median Absolute Deviation (MAD); the upper one is the 95th percentile (AD95). For clarity, there is a slight horizontal shift between the MAD and AD95 symbols. The bottom and top edges of each box indicate the 25 th and 75th percentiles within the corresponding data set; the horizontal line is the median value; whiskers extend to the extreme lower and upper values. Regression curves are drawn: a solid line through the MADs, a dotted line through the AD95s. All magnitude values are given in unit of 0.001 . See text for more explanation

dramatically improves when three or more frames have been obtained in stable photometric conditions. Figure 5 shows that the $\sigma_{\mathrm{fr}}\left(g_{0}\right)$ during a stable period governs the accuracy of the resulting calibration. The additional $n_{\mathrm{fr}}-n_{\mathrm{fr}}\left(g_{0}\right)$ frames may even be obtained in very bad conditions (in the case of Fig. $4 \sigma_{\mathrm{fr}}=0.032$ ). Figure 6 illustrates the role of these additional frames - each of them being an entire group. It is certainly advantageous to increase their number. The value of AD95 decreases from 0.013 down to 0.008 thanks to the inclusion of 10 "bad" frames.

Figures 7 and 8 show the effect of simply increasing the number of frames, under good photometric conditions
( $\sigma_{\mathrm{s}}=0.004$ or 0.008 and $\sigma_{\text {fr }}=0.004$ ), in a single group. As expected, the accuracy rapidly improves until about $n_{\text {fr }} \sim 10$. Piling up more frames may be interesting when the accuracy of the measurements, $\sigma_{\mathrm{s}}$, is not perfect (faint stars).

It can be seen from Fig. 9 that very large number of stars are not needed under usual conditions. They may be necessary when much more complex models are adopted for $z_{\mathrm{ff}}$.

Figure 10 shows the degradation occuring when fainter stars - i.e., with $\sigma_{\mathrm{s}, 2}$ significantly larger than $\sigma_{\mathrm{s}, 1}$ - are included. A single group of 16 frames is considered. 
Figure 11 illustrates that large inter-group shifts are of no consequence. Groups of frames obtained at different air masses, even on different nights can be mixed at will, provided a unique flat-field calibration remains valid.

The spatial repartition of the frames in the special, high-quality, group $g_{0}$ is of little importance. The groups $g_{0}$ corresponding to Fig. 12 contain three frames obtained with offsets in different directions. The "area" in abscissa is the external product of these offsets; the unit is $(\delta x)^{2}=$ $(\delta y)^{2}$. Of course, extremely small or large offsets must be avoided, as well as parallel offsets.

\subsection{Standard stars}

Figures 13 to 21 present results when stars have accurately known values. We suppose here that these values are perfectly known: i) if they were not we would fall back to the case of no standards; ii) repeated observations of the same few clusters can easily lower the errors on the instrumental magnitudes below a few 0.001 mag.

It is obvious from Figs. 13 to 21 that good instrumental standards allow a much better accuracy to be reached with fewer observations.

Figures 13-14 show that even bad observations can yield a correct flat-field calibration: 16 frames with an inter-frame $\sigma_{\mathrm{fr}}$ as large as 0.032 and a measurement noise $\sigma_{\mathrm{s}}=0.020$ yield an accuracy of about $0.010 \mathrm{mag}$. The effect of $\sigma_{\mathrm{fr}}$ can be alleviated by disconnecting all zero points. This is barely noticeable in Fig. $15\left(\sigma_{\mathrm{fr}}=0.004\right)$, but quite obvious in Fig. $16\left(\sigma_{\mathrm{fr}}=0.032\right)$. This procedure must be adopted when observations are carried out in poor weather. The importance of standards in bad photometric conditions is clearly shown by comparing Figs. 4 and 16 . The accurate correction of zero-point shifts, however large they can be, is also examplified by Fig. 21.

A single exposure can be used if enough standard stars are available: $n_{\mathrm{fr}}$ frames with $n_{\mathrm{s}}$ stars are about (but not quite) equivalent to a single frame with $n_{\mathrm{fr}} \times n_{\mathrm{s}}$ stars. The case of Fig. 17 for $n_{\mathrm{fr}}=1$ is rather extreme (an average of only 22 measurements for 10 parameters), but even these poor data yield an accuracy better than $0.010 \mathrm{mag}$. A larger $n_{\mathrm{s}}$ is a definite advantage as shown in Fig. 18 which assumes a stellar density four times higher. This is also illustrated by Fig. 19. Calibration errors can be significantly lower than $\sigma_{\mathrm{s}}$.

\section{Conclusions}

Numerical simulations give quantitative estimates of the various errors affecting the stellar calibration of CCD flat fielding. More precise guidelines can then be given on how perform this procedure.

- Accurate flat-field calibration using the stellar method (Paper I) is perfectly possible under non-photometric conditions - even very bad ones - by using cluster standards.
- If no such standards are available, the stellar method is still possible if three exposures can be secured in a period of very stable conditions. Since these exposures can be obtained on a single frame, the required period of stability can be very short, e.g., less than one minute. More generally, there should be at least two pairs of exposures (possibly in different, but highquality, groups) with non-parallel offsets.

- The quality of the flat-field calibration is always improved by securing additional frames, even in nonphotometric conditions, and even on different nights (if the instrumentation is stable).

The stellar fields needed for flat-field calibration must be moderately crowded, e.g., a minimum of 10 or 20 stars per frame (if a dozen exposures are obtained), or 50 to 100 stars per frame (for only one or two exposures). If the fields are not too crowded, multiple exposures can be taken on single frames, allowing a faster observational procedure.

As a rule, we use to make several multiple exposures (with spatial offsets), and we bracket them with short series of single exposures at the same position as a check of the stability of the transparency, and to select the best $g_{0}$ groups. Each series is done at a different position, so these frames are also useful in the overall procedure. The calibration procedure can be carried out over several fields (same parameters $\boldsymbol{b}$ ), and can be smoothly integrated within the extinction measurements.

Needless to say, variable stars must be checked out, and the possible need of accurate color transformation may impose additional constraints on the diversity of stellar types and reddenings. A dozen carefully selected fields could adequately cover an hemisphere and provide the necessary data for calibrating the detector as well as for carrying out global reduction procedures (Manfroid 1993).

The observational procedure is simple and the gain in accuracy considerable, if very accurate cluster standards exist in the instrumental system. This is almost never the case, and observers use to accept a considerable loss in calibration accuracy, and/or a loss in science observing time. Days or weeks spent in setting up such standards are not wasted. They would rapidly pay back in terms of increased efficiency and accuracy. A wise long-term observational and reduction strategy leads to a steady increase in the quality of the standards and, basically, of all reduction parameters (extinction, etc.). However, nobody would like to wait months or years before getting proper reductions. Thus, a special observational effort should be done on the standards as soon as a new system is installed, in order to be able to produce rapidly high-quality data.

The present results refer to the monochromatic case. To estimate color effects in the heterochromatic case, one only needs a larger star number, a wide range of colors, and a realistic model for the color-sensitivity index (Paper I) in order to minimize the number of free parameters. These issues will be discussed in a forthcoming paper. 
Acknowledgements. The computations have been performed on computers at the SEGI and at the Institut d'astrophysique (University of Liège), partly thanks to contracts ARC94/99178 ARCCF (Belgium), and SC005 SCRN initiated by the Belgian State, Science policy programming under SSTC. Partial support through the PRODEX XMM-OM project is also gratefully acknowledged.

\section{References}

Manfroid J., 1993, A\&A 271, 714

Manfroid J., 1995, A\&AS 113, 587

Tobin W., 1993, in: Stellar photometry - Current techniques and future developments, IAU Coll. 136. In: Butler C.J. \& Elliott I. (eds.). Cambridge University Press, p. 304 